\title{
The Empirical Study of Trajectory-based Tasks in Pen+touch Interfaces
}

\author{
Jibin Yin ${ }^{\mathrm{a}}$, Hui Yang ${ }^{\mathrm{b}}$, Yang Gao ${ }^{\mathrm{c}}$ and Bo Zhang ${ }^{\mathrm{d}}$ \\ Kunming University of Science and Technology, Kunming 650000, China \\ ajyb@cnlab.net, byanghui@cnlab.net, 'cgaoyang@cnlab.net, ${ }^{\mathrm{d}}$ zhangbo@cnlab.net
}

Keywords: Trajectory-based task, pen+touch, interface design.

\begin{abstract}
Trajectory-based tasks are quite common in pen+touch interfaces. However, there is a lack on empirical knowledge of directly using fingers to perform trajectory-based tasks in the steering law paradigm. In this study we investigate the human ability of using fingers to perform trajectory-based tasks in pen+touch interfaces. A controlled experiment is designed and conducted, involving the $\mathrm{DH}$ and NDH manners. The experiment shows that when using fingers to perform steering tasks the minimum width of steering task tunnel is about 60 pixels. When using the dominant (DH) or non-dominant hand (NDH) to conduct steering tasks there is no significant difference between the two manners for the steering time and the error rate. The current work provides the empirical knowledge as basis for future research and design of finger performance applications.
\end{abstract}

\section{Introduction}

Pen+touch interaction is an attractive user-computer interface paradigm, which combines the advantages of the pen writes and touch manipulations. With the advances in hardware technology, computing in the forms of handheld devices, tablets, iPhones and iPads has made pen+touch interfaces increasingly more relevant to mainstream applications. Consequently, research into pen+touch interaction methods has intensified in recent years [1]. In essence, pen+touch performances belong to two-handed interactions. The pen provides precise input (due to the thin pen-tip) while the finger touch supports rough input (the thick finger-tip). However, the touch input is more flexible than the pen input.

Trajectory-based tasks are quite common in pen+touch interfaces. It requires users to draw strokes within a specified steering tunnel. One model and paradigm used for studying trajectory-based human-computer interaction tasks is the steering law [2] akin to Fitts' law for target pointing tasks. The steering law quantifies the difficulty of a trajectory task with an index, and relates path steering time with the index in a linear fashion. The steering law has been verified with several input devices, such as the stylus, mouse, isometric joystick, touchpad, and trackball [3], and in different movement scales [4]. Other researchers have studied and applied the steering law in a variety of settings [5, 6]. For example, recently Pastel [5] studied steering through paths with corners, and found that some corner angles were more difficult than others. Steering law has been used in various scenarios, however, there is a lack on empirical knowledge of directly using fingers to perform trajectory-based tasks in steering law paradigm. When using fingers to directly perform steering tasks, some special problems need to be considered such as the width of steering tunnel, the dominant hand (DH) and the non-dominant hand (NDH). Clearly, the steering law can potentially offer a systematic evaluation paradigm for studying properties of finger input in steering tasks, which to our knowledge has never been done. Our current study focuses on it. Common basic questions regarding humans' ability of using fingers to perform trajectory-based tasks arise. For example:

When using fingers to perform trajectory-based tasks, what is the minimum width of steering task tunnel?

What effects can users' performance hand (dominant and non-dominant hands) bring in manipulating trajectory-based tasks?

The goal of our study is to answer these fundamental and general questions systematically and empirically. An experiment is designed and conducted to reveal the questions. Finally, we discuss and 
make conclusions of our findings.

\section{Experiment}

This study focuses on the human ability of using fingers to directly perform trajectory-based tasks. Generally, a finger-tip of a user has bigger size than a pen-tip so the finger input is not quite precise. When directly using a finger to perform a steering task we should first investigate the minimal width of steering task tunnel. This is the main goal of the experiment. When using the dominant hand (thereafter referred to as $\mathrm{DH}$ ) or non-dominant hand (thereafter referred to as NDH) to perform steering tasks the minimal width of steering task tunnel may differ, which is also examined by our experiment.

Our experiment, therefore, consisted of two parts. Part 1 measured the distribution of users' finger-tip physical size. Part 2 measured the minimal width of the steering task tunnel in DH or NDH manner.

Part 1.

Participants and Apparatus. Fifteen volunteers ( 9 males and 6 female) participated in Part 1 of the experiment. The average age was 26 years (ranging from 23 to 33). All were right-handed. The Mahr's digital vernier caliper was utilized to measure the width of a participant' forefinger. Its precision is $0.01 \mathrm{~mm}$.

Procedure and Design. In the following experiments, participants used forefinger to touch a screen to perform steering tasks. Therefore, we measured the width of the participant's forefinger in Part 1 . We measured the forefinger of the $\mathrm{DH}$ and $\mathrm{NDH}$, and the accuracy of the measured results is set at $0.1 \mathrm{~mm}$.

Results. Fig. 1 was the distribution of forefinger's width around their average value. The minimum width of the DH forefinger is $12.8 \mathrm{~mm}$, the maximum width is $17.6 \mathrm{~mm}$. The minimum width of the NDH forefinger is $12.2 \mathrm{~mm}$, the maximum width is $17.1 \mathrm{~mm}$. The average width of the $\mathrm{DH}$ forefinger is $15.5 \mathrm{~mm}$, and that of the NDH finger is $15.3 \mathrm{~mm}$. The difference between them is $0.2 \mathrm{~mm}$. When setting the width of a steering tunnel, we ignored the width difference between of the $\mathrm{DH}$ forefinger and the NDH forefinger.
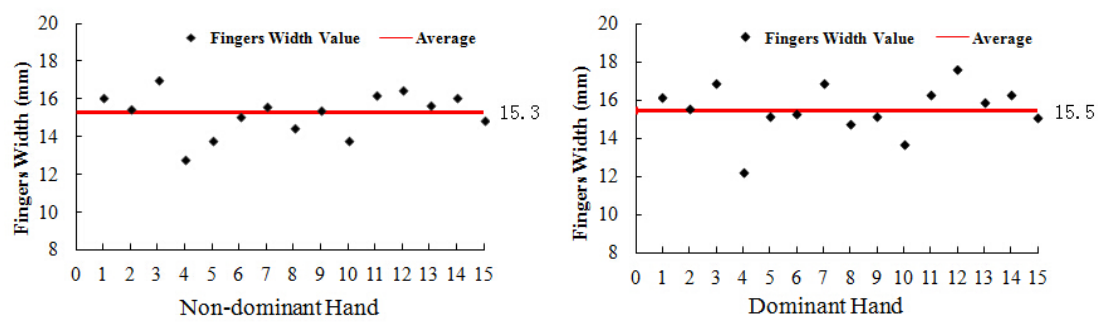

Fig. 1 Distribution of forefinger's width around the average value

\section{Part 2.}

Participants and Apparatus. The participants recruited in Part 2 were the same to those of Part 1. The experimental interface was run in an Apple iPad mini 2 with a $200 \times 134.7 \mathrm{~mm}$ retina display. Its operating system is IOS 6. Its resolution is 2048x1536 pixels and it supported multi-touch. The experimental program was written in Objective $\mathrm{C}$ using Adonit Jot Touch SDK (the Adonit Jot Touch SDK is a free framework for the IOS).

Task and Stimuli. There are two types of tasks in Part 2: linear tasks and circular tasks. The tasks (see Fig. 2) were based on the steering law.

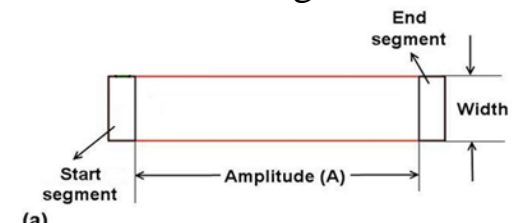

(a)

(b)

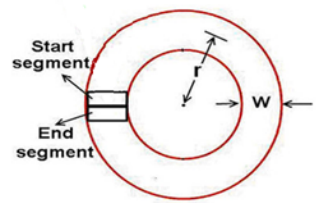

Fig. 2 Experimental tasks in Part 2: (a) linear tasks; (b) circular tasks

The basic formulation of the steering law is the following: 


$$
\begin{aligned}
& T=a+b I D \\
& I D=\int_{C} \frac{d s}{W(s)}
\end{aligned}
$$

Where $T$ is the time to successfully steer through path $C$ and $W(s)$ is the path width at $s$. ID is the index of difficulty. $a$ and $b$ are constants. The current experiment used two types of path: linear and circular. For a linear path with constant width $W$, (2) can be simplified to:

$$
I D=\frac{A}{W}
$$

Where $A$ is the path length.

For a circular path with constant width $W$, (1) can be simplified to:

$$
I D=\frac{2 \pi r}{W}
$$

Where $r$ is the radius of the circular path.

$a$ and $b$ change with the steering device, type of path, etc., and hence can serve as performance indicators.

In this experiment, there were a start segment and an end segment on the two ends of each steering path. The participants first placed a finer-tip on the start segment. The participants proceeded to draw a red line on the screen, displaying the finger's trajectory. The steering timer started when the finger-tip left the start segment and ended when the finger-tip entered the end segment. An error was recorded and the trial was abandoned if the finger-tip steered outside of the borders of the path. The participants were asked to minimize errors.
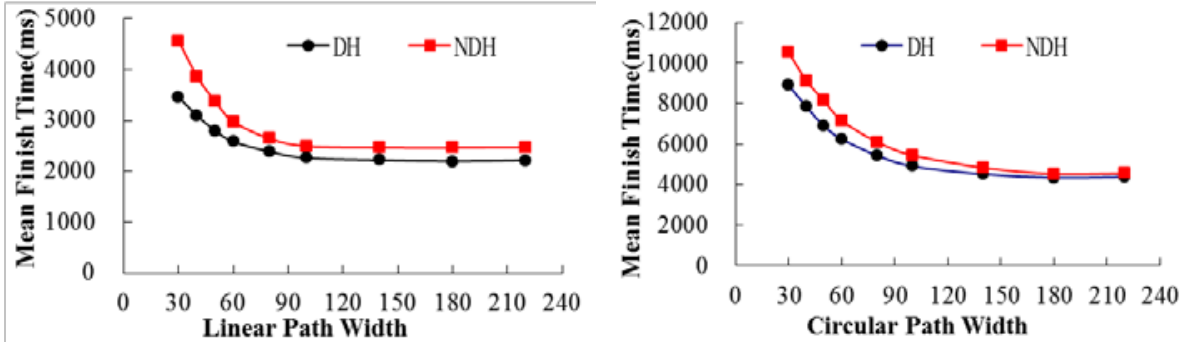

Fig. 3. The mean steering time for tasks with different tunnel widths at DH and NDH manners

Procedure and Design. A within-participants factorial design with repeated measures was used. The independent variables were the following: path type (linear and circular), path length ( $\mathrm{A}=300$ pixels), and path width (W= 30, 40, 50, 60, 80, 100, 140, 180 and 220 pixels). The dependent variables were the steering time and error rate. For each combination of the independent variables, two trials were performed. The total number of trials was 2 (types of steering tasks) $\mathrm{x} 1$ (length) $\mathrm{x} 9$ (width) $\times 2(\mathrm{DH}$ and $\mathrm{NDH}) \times 3$ (repetitions) = 108. In the beginning of the experiment, 20 trials ranging from the easiest to most difficult conditions (A and $\mathrm{W}$ ) in both linear and circular paths were run as practice trials. The total duration of the experiment was about 30 minutes. Whenever the participants felt tired, they were allowed to take a break.

Results. Steering Time: Fig. 3 showed the mean steering time for tasks with different path widths. It could be observed from Fig. 3 that the mean steering time of NDH is slightly longer than that of DH for tasks with the same path width. With the decrease of path widths, the difficulty of steering tasks increases gradually and its steering time will increase too. When the width of tunnel exceed 60 pixels the steering time changed gradually. When the widths were less than 60 pixels the steering time increases sharply.

Error Rate: In the experiment, if a trial went out of the boundaries of the path before completion of a trial, an error was recorded and the trial was repeated. For the linear steering tasks at DH manner the error rates (width ranged from 30 to 220 pixels) were 17.9\%, 9.7\%, 8.1\%, 5.1\%, 2.8\%, 1,9\%, 0.8\%, $0.6 \%$ and $0.4 \%$, respectively. For the linear steering tasks at NDH manner the error rates (width ranged from 30 to 220 pixels) were $31.2 \%, 18.6 \%, 12.5 \%, 8.7 \%, 5.1 \%, 2,3 \%, 1.3 \%, 0.8 \%$ and $0.2 \%$, respectively. For the circular steering tasks at DH manner the error rates (width ranged from 30 to 220 pixels) were $43.2 \%, 24.6 \%, 13.3 \%, 7.7 \%, 5.8 \%, 2,4 \%, 1.2 \%, 0.0 \%$ and $0.0 \%$, respectively. For the 
circular steering tasks at $\mathrm{NDH}$ manner the error rates (width ranged from 30 to 220 pixels) were 81.3\%, 58.5\%, 30.6\%, 19.5\%, 10.6\%, 7,4\%, 3.2\%, 0.9\% and 0.0\%, respectively.
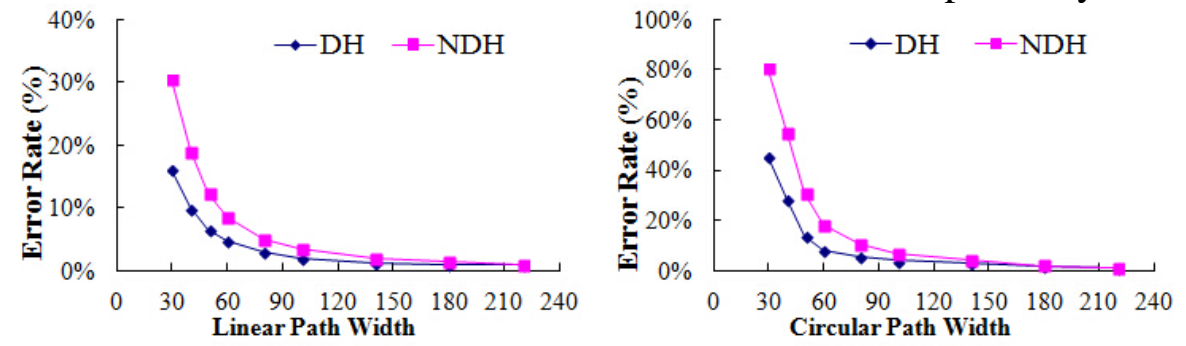

Fig. 4 The error rate for tasks with different tunnel widths at DH and NDH manners

When the width of steering path was less than 60 pixels, according to Fig. 4, the error rate changed rapidly, especially for NDH manner of circular tasks. The experimental results show that the tunnels with the width which is more than 60 pixels are easy to perform.

\section{Summary}

In this study we investigate the human ability of using fingers to perform trajectory-based tasks in pen+touch interfaces. A controlled experiment is designed and conducted, involving the $\mathrm{DH}$ and $\mathrm{NDH}$ manners. The experiment indicates that the minimum width of the $\mathrm{DH}$ forefinger is $12.8 \mathrm{~mm}$, the maximum width is $17.6 \mathrm{~mm}$. The minimum width of the $\mathrm{NDH}$ forefinger is $12.2 \mathrm{~mm}$, the maximum width is $17.1 \mathrm{~mm}$. The average width of the DH forefinger is $15.5 \mathrm{~mm}$, and that of the NDH finger is $15.3 \mathrm{~mm}$. The experiment also shows that when using fingers to perform steering tasks the minimum width of steering task tunnel is about 60 pixels. When using the dominant or non-dominant hand to conduct steering tasks there is no significant difference between the two manners for the steering time and the error rate. The empirical knowledge provides guidelines for touch-based UI designs.

\section{Acknowledgements}

This study is supported by the National Natural Science Foundation of China (Grant No. 61262042).

\section{References}

[1] Brandl, P., Forlines, C., Wigdor, D., Haller, M. and Shen, C. Combining and measuring the benefits of bimanual pen and direct-touch interaction on horizontal interfaces. AVI '08 Conf. on Advanced Visual interfaces. pp. 154-61, 2008.

[2] Accot, J. and Zhai, S.,1997. Beyond Fitts' Law: Models for Trajectory-Based HCI Tasks. In: Proceedings of the Conference on Human Factors in Computing Systems, CHI'97, 295-302.

[3] Accot, J. and Zhai, S, 1999. Performance Evaluations of Input Devices in Trajectory-based Tasks: An application of the Steering Law. In: Proceedings of the Conference on Human Factors in Computing Systems, CHI'99, 466-472.

[4] Accot, J., and Zhai, S, 2001. Scale Effects in Steering Law Tasks. In: Proceedings of the Conference on Human Factors in Computing Systems, CHI2001, 1-8.

[5] Pastel, R, 2006. Measuring the Difficulty of Steering Through Corners. In: Proceedings of the Conference on Human Factors in Computing Systems, CHI2006, 1087-1096.

[6] Tudoreanu, M., and Kraemer, E, 2002. A Study of the Performance of Steering Tasks under Spatial Transformation of Input. In submission to Joint EUROGRAPHICS - IEEE TCVG Symposium on Visualization. 